\title{
Adult cancer patients' barriers toward pain management: A literature review
}

\author{
Hodan Abdullah*1, Fadi Khraim ${ }^{2}$, Atef Al-Tawafsheh ${ }^{1}$ \\ ${ }^{1}$ Hamad Medical Corporation, Doha, Qatar \\ ${ }^{2}$ University of Calgary in Qatar, Doha, Qatar
}

Received: May 28, 2020

DOI: $10.5430 /$ jnep.v10n12p38
Accepted: July 28, 2020

URL: https://doi.org/10.5430/jnep.v10n12p38

\begin{abstract}
Background and objective: Cancer pain is the most common symptom among cancer patients. Despite strategies to control cancer pain, cancer patients' beliefs and attitudes influence the effectiveness of cancer pain management. The aim of this literature review was to identify and explore adult cancer patients' barriers toward pain management.

Methods: A literature review was conducted. CINAHL, Medline, and PsychINFO databases were searched for relevant articles from 2008 to 2019. Twenty one articles were included in this literature review. Thematic analysis was conducted to identify and explore adult cancer patients' barriers toward pain management.

Results: This literature review revealed several patient barriers toward pain management. These barriers were categorized into cognitive barriers that include poor pain communication, fatalism, and fear of addiction and tolerance; sensory barrier, such as fear of drug side effects; affective barriers, such as anxiety and depression, and socio-demographic barriers that influence cancer pain management.

Conclusions: Adult cancer patients' barriers toward pain management significantly compromise the effectiveness of pain management and affect cancer patients' quality of life. A better understanding of cancer patients' barriers toward pain management by healthcare providers will result in better assessment and management of these barriers and will enhance evidenced-based patient education.
\end{abstract}

Key Words: Cancer pain, Cancer patient, Barrier, Pain management, Pain control

\section{INTRODUCTION}

Cancer is a worldwide burden that causes morbidity and mortality. In 2018, World Health Organization (WHO) reported that there were 9.6 million cancer-related deaths. ${ }^{[1]}$ This burden raised an alarm globally to develop strategies to prevent cancer, provide evidence-based treatment, and manage cancer-related symptoms. Cancer pain is one of the most common symptoms among cancer patients and ranges in prevalence from 14 to $100 \%{ }^{[2]} \mathrm{Kim}$, et al. ${ }^{[3]}$ and Rau et al. ${ }^{[4]}$ stated that cancer pain occurs in all stages with an estimated prevalence of $25 \%$ for newly diagnosed patients, $33 \%$ for those undergoing active treatment, and around $75 \%$ for those with advanced disease. Pergolizzi et al., ${ }^{[5]}$ surveyed 5,084 European patients with cancer pain and reported that $44 \%$ of respondents experienced severe cancer pain. Of all types of cancer pain, $64 \%$ experienced neuropathic pain, ${ }^{[5]}$ while $59.2 \%$ suffered from breakthrough pain. ${ }^{[6]}$

Pain is not only a result of a biological mechanism; it is a complex perceptual experience. The gate control theory of Melzack and Wall pinpointed that input modulation by

*Correspondence: Hodan Abdullah; Email: HIbrahim7@ hamad.qa; Address: Hamad Medical Corporation, Doha, Qatar. 
patients' emotions and cognitions defines the pain experience. ${ }^{[7]}$ Cancer pain is a subjective and the individualized experience of pain is shaped by the interactions of multiple factors including physiological, psychological, social, cultural, behavioral, cognitive, and sensory aspects of cancer patients. Patients' expectations, beliefs, and hope contribute to the individualize meaning of cancer pain. ${ }^{[8]}$ Different religious beliefs are also core elements to the experience of cancer pain. Some cancer patients perceive cancer pain as a reward and an indicator of life, while for others, cancer pain is a sign of disease progression, metastasis, or end-of-life. ${ }^{[9]}$ A variety of perceptions and beliefs contribute to the experience of cancer pain and may act as barriers toward adequate pain management; ${ }^{[8,9]}$ accordingly, health care providers should consider patient individualized experience while managing cancer pain.

Because cancer pain has serious impacts on patients' lives, it is important to eliminate any patients' barriers that impede the effectiveness of pain management. Cancer pain affects their physical, social, psychological, and financial wellbeing. ${ }^{[10]}$ It may compromise their functions and activities of daily living, reduce their family roles, and may lead to social isolation. It may also lead to sleep disturbance, affect patients' interpersonal relationships and reduce enjoyment of life. ${ }^{[7]}$ Depression and changes in mood are among the most significant psychological symptoms that cancer patients may experience due to pain. ${ }^{[4]}$ These symptoms may reduce cancer patients' quality of life, contribute to poor adherence to treatments, and produce suicidal thoughts. ${ }^{[4]}$ Rouahi and Zouhdi ${ }^{[10]}$ stated that cancer patients with pain may experience additional financial burdens related to cancer pain treatments' costs or compromised job security due to absence from work. This might become an additional barrier that can reduce patients' adherence to cancer pain treatment.

Pain is a significant symptom in cancer patients that has serious complications. Therefore, it is crucial to promote effective cancer pain management strategies and overcome patients' barriers. These strategies will help health care providers practice their ethical duty to relieve pain, reduce patients' misconceptions about analgesics, protect cancer patients' dignity, and maintain patients' human right. Therefore, the WHO created cancer pain ladder, which is a universal pain management strategy that consists of three steps to control cancer pain for adult patients (see Figure 1). The ladder contains explanation of orders of administering pain medication immediately when cancer pain occurs. ${ }^{[5]}$ The WHO's guideline was reported to be $86 \%$ to $100 \%$ effective in treating severe cancer pain. ${ }^{[11]}$ This indicates high degree of effectiveness of WHO pain relief ladder as a strategy to treat cancer pain when patients adhere to pain medications.

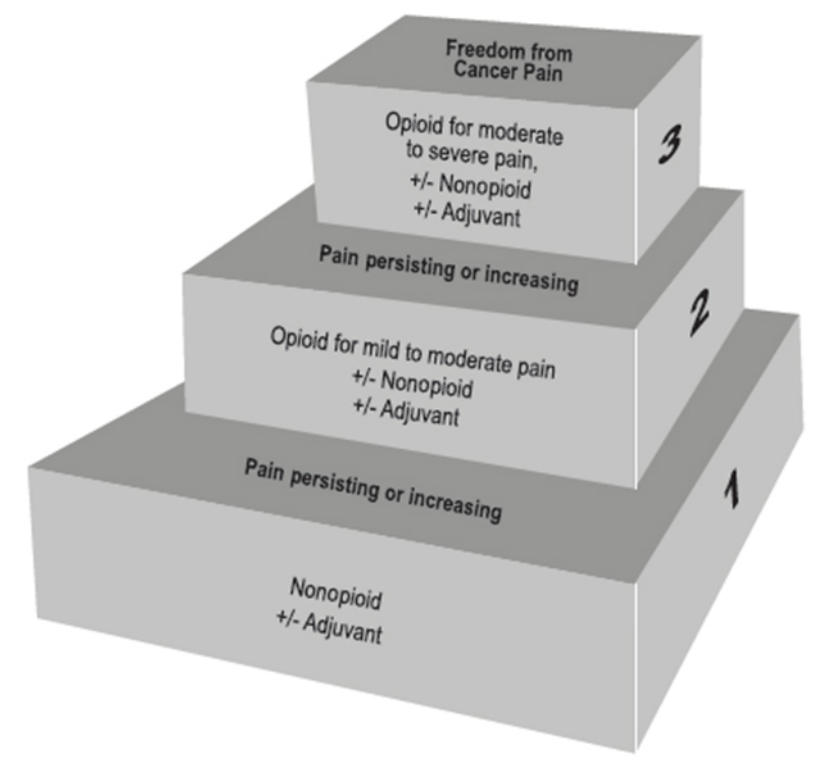

Figure 1. World Health Organization's pain relief ladder

Vital collaboration among all stakeholders is required to enhance the effectiveness of cancer pain management and prevent barriers of proper pain management among this patient population. The knowledge of health care professionals related to analgesics and their ability to facilitate patients' access to these analgesics are important to achieve effective cancer pain management. ${ }^{[4]}$ Oncologists and oncology nurses must also have proper communication skills with cancer patients in order to assess their pain and beliefs about pain management. ${ }^{[10]}$ Furthermore, it is important, as a first step to effective cancer pain management, that health care professionals utilize reliable, valid, and comprehensive pain assessment tools.

Effective cancer pain management can be hindered by patients, organizational and health care providers' barriers. Organizational barriers consist of lack of financial resources and access to pain analgesics in some countries. ${ }^{[1]}$ Excessive rules and regulations for the administration of opioids is another barrier that contributes to under-treatment of cancer pain. ${ }^{[12]}$ Misconceptions about opioids, poor knowledge about cancer pain management, and negative attitude towards cancer, and cancer pain are the most common barriers among health care providers. ${ }^{[12]}$ Cancer patients' barriers have been studied well in many contexts. A systematic review conducted by Jacobsen et al. ${ }^{[13]}$ in 2009 categorized these barriers into cognitive, sensory, and affective barriers. They found that cognitive barriers consist of patients' beliefs, attitudes, and concerns about analgesics; sensory barrier was related to the opioids' side effects; and the affective barriers described cancer patients' emotional factors such as depression and anxiety. ${ }^{[13]}$ Another recent systematic review by 
Makhlouf et al. ${ }^{[14]}$ in 2020 reported that lack of knowledge and negative attitudes of cancer patients were the key factors for their attitudinal barriers toward cancer pain management. However, there is a deficit in the literature in identifying different cancer patients' barriers as well as the interconnection among those barriers. Therefore, the purpose of this paper was to conduct a literature review to identify and explore adult cancer patients' barriers toward pain management that will enhance enhance effective cancer pain management and positively influence patients' quality of life and functional ability or help them to die with dignity.

\section{Methodology}

A literature review was conducted using the framework described by Cronin and colleagues. ${ }^{[15]}$ The keywords that were used to search databases included: cancer pain, cancer patient or patient*, oncology patient*, barrier*, attitude or attitud*, pain control*, pain management, palliative care, self-report*, pain report*, and patient* report*. Cumulative Index to Nursing and Allied Health Literature (CINAHL), Medline, and PsychINFO databases were searched for rel- evant articles. The initial search identified a total of 2,736 articles. Peer-reviewed, English language, adult population, and original research articles published between 2008 until 2019 were used as limiters. After applying the limiters, 821 articles were identified for additional screening and possible inclusion. The title and abstract screening were conducted to assess the articles for their relevance to the research question. Articles related to caregivers and health care provider's experiences or patients' perceptions of cancer pain were excluded. The screening resulted in excluding 765 articles. A total of 56 articles were identified for possible inclusion. After removing the duplicates $(n=15)$, the remaining 41 articles were subjected to full text review.

The full text review of the remaining 41 articles eliminated another 20 articles because these articles did not examine patients' barriers. A total of 21 articles were determined to be suitable for inclusion in this literature review; 19 articles were quantitative studies and two were qualitative studies. PRISMA chart summarizes the search process (see Figure 2). ${ }^{[16]}$
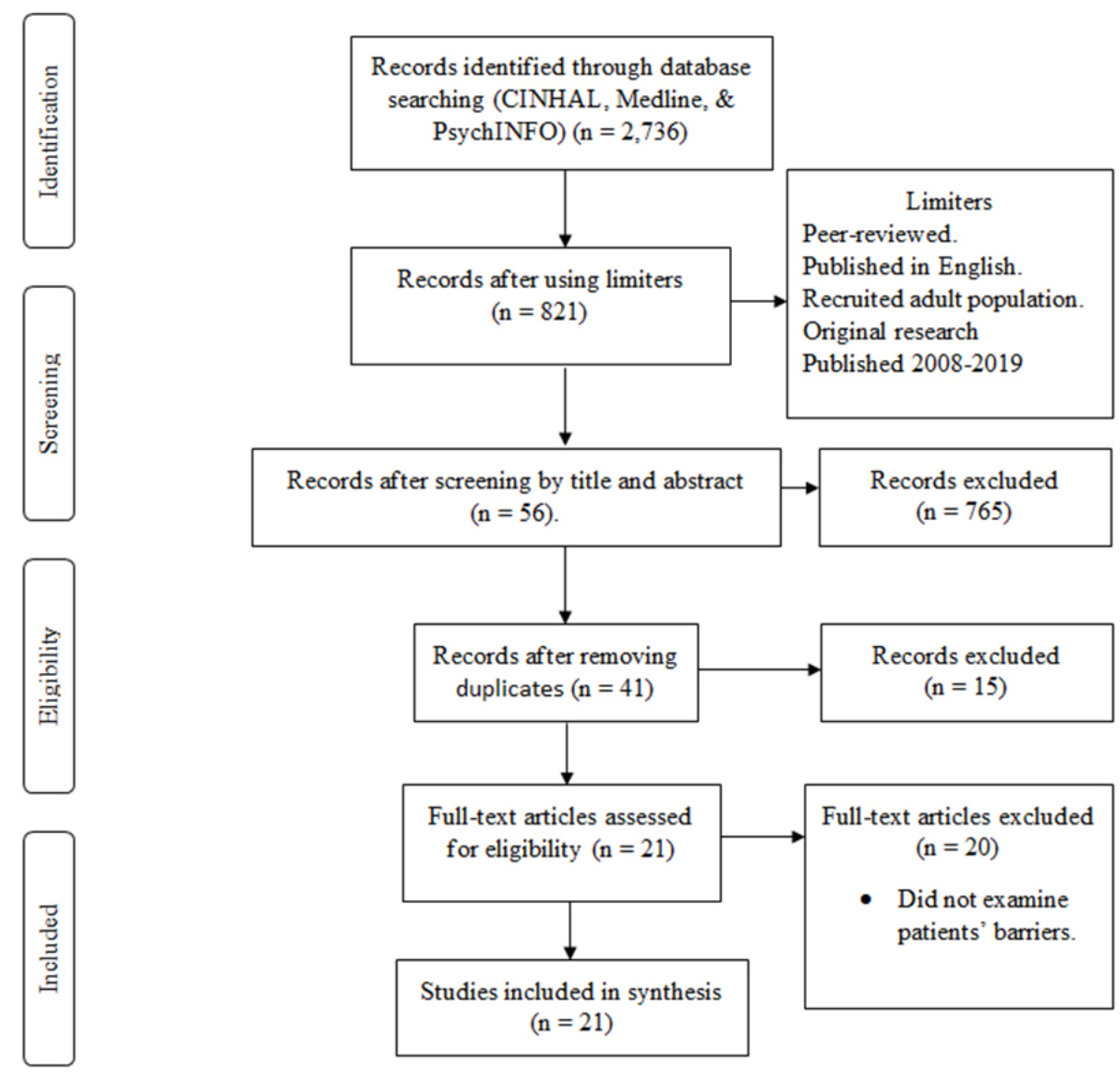

Figure 2. PRISMA chart of the search process 
The Mixed Method Appraisal Tool (MMAT) ${ }^{[17]}$ was used to examine the quality of the 21 remaining articles. All articles were deemed to be of adequate quality based on the MMAT criteria, therefore all were included in the literature review. Thematic analysis as described by Braun et al. ${ }^{[18]}$ was conducted using a summary table. We considered careful and deep reading while findings were extracted from these articles in order to identify similar themes. The main themes that emerged from selected articles were poor pain communication, fatalism, fear of physiological dependence, fear of drug side effects, affective barriers, and socio-demographic factors.

\section{Results}

The aim of the literature review was to identify and explore adult cancer patients' barriers toward pain management. Six main themes emerged from the 21 retrieved articles. These themes were poor pain communication, fatalism, fear of physiological dependence, fear of drug side effects, affective barriers, and socio-demographic factors. The themes are described below.

\subsection{Poor pain communication}

Effective therapeutic communication between patients and health care providers is essential to perform adequate pain assessment and proper pain management. However, negative beliefs that cancer patients have related to cancer pain may influence the effectiveness of communication in pain management. Jacobsen et al., ${ }^{[19]}$ examined the barriers of 30 Lithuanian cancer patients; and they found that communication barriers include beliefs that good patients do not complain, and that discussing pain may distract physicians from treating cancer. Misconceptions about pain communication have been reported to be a common barriers among cancer patients. ${ }^{[19-21]}$ These misconceptions about communicating cancer pain were examined by Edrington et al. ${ }^{[21]}$ in a sample of 50 Chinese Americans, and explored qualitatively by Liu et al. ${ }^{[2]}$ in a sample of nine cancer patients from China. Participants in both studies did not report their cancer pain, and showed desire to be good patients and unwillingness to distract physicians. A study conducted on 300 Jordanians cancer patients reported similar results. ${ }^{[23]}$ More than half of the participants believed that being good patient means not to report pain and third of participants did not report pain because they did not want to distract physicians from treating cancer. In contrast, poor communication as a barrier to manage cancer pain was one of the least concerns among other barriers for 196 American $^{[24]}$ and 183 Taiwanese cancer patients. ${ }^{[25]}$

Published by Sciedu Press

\subsection{Fatalism}

Fatalism is another barrier that influences the effectiveness of cancer pain management. Some patients have beliefs, such as cancer pain is inevitable or cancer pain is from God. ${ }^{[22,26]}$ These beliefs contribute in reducing the effectiveness of cancer pain management. Kwon et al. ${ }^{[27]}$ reported in a quantitative study that Korean cancer patients $(n=201)$ had less fatalistic beliefs; however, those participants with fatalistic beliefs had significantly less pain relief. Other studies conducted in some European countries, such as Norway, Germany, and Iceland, reported lower scores of fatalistic thoughts among participants. ${ }^{[28]}$ On the other hand, Haozous et al. ${ }^{[29]}$ in their ethnographic qualitative study found American Indians strongly believe that cancer pain is inevitable. The beliefs that pain is inevitable and cancer pain is from God were considered the main barriers among most American cancer patients who belonged to minority groups. ${ }^{[20,30]}$ Fatalistic beliefs were also reported among Chinese Americans who live alone ${ }^{[28]}$ or among Lithuanian cancer patients who live in villages. ${ }^{[19]}$ Saifan et al. ${ }^{[23]}$ reported that Jordanian cancer patients had more fatalistic beliefs than what is reported in studies from other countries.

\subsection{Fear of physiological dependence}

Addiction and tolerance of using analgesics are two concerns of cancer patients toward pain management. Alexopoulos et al. ${ }^{[31]}$ studied the experience of 134 Greek cancer patients at eight oncologic centers, and found that $61 \%$ of study participants were reluctant to adhere to cancer pain medication because of their concerns of addiction to painkillers. Similar findings were reported in a study with large, heterogeneous, random sample of Americans $(n=4,707)$ that addiction concern was high among participants. ${ }^{[30]}$ Furthermore, Valeberg et al. ${ }^{[32]}$ reported positive significant relationship between fear of tolerance and poor pain relief among 71 Norwegian cancer patients. In a study conducted in South Korean outpatient palliative care on 201 cancer patients, concerns of addiction and tolerance were the highest among other barriers toward cancer pain management. As a result, study participants did not report pain and refused cancer pain management. ${ }^{[27]}$ Two studies on Jordanians cancer patients who were prescribed analgesics for management of cancer pain reported relatively similar results. ${ }^{[23,33]}$ While Saifan et al. ${ }^{[23]}$ reported three quarters of participants have concerns of addiction and tolerance, Al Atiyyat et al. ${ }^{[33]}$ found moderate level of these concerns in their study participants. Addiction and tolerance were also deemed impediments for effective cancer pain management in other studies. ${ }^{[22,26,34-36]}$ 


\subsection{Fear of Drug Side Effects}

Cancer pain analgesics, especially strong opioids, produce side effects that cancer patients find unpleasant. Most frequently reported side effects were nausea, drowsiness, and saying or doing embarrassing things. ${ }^{[23,30,34,36]}$ This barrier was considered the second highest barrier among Norwegian and Jordanian cancer patients who are in pain. ${ }^{[28,37]}$ Haozous et al. ${ }^{[34]}$ found 13 Indian Americans who participated in their study constantly link their poor adherence to cancer pain management to their fear of doing embarrassing things. According to Liang et al., ${ }^{[34]}$ approximately $59 \%$ of 92 Taiwanese patients in their study indicated cancer pain medication's side effects as a barrier toward effective pain management. Similarly, in a study on multiethnic American participants, fear of analgesics' side effects was found to be a significant barrier to effective cancer pain management. ${ }^{[30]}$ In contrast, a moderate concerns of drug side effects were found in a comparison study between Germany, Iceland, and Norway. ${ }^{[28]}$

\subsection{Affective Barriers}

Anxiety and depression are two common affective barriers of cancer pain management. In a comparative study between Lithuanian $(\mathrm{n}=30)$ and Danish $(\mathrm{n}=33)$ cancer patients, Jacobsen et al. ${ }^{[37]}$ reported a significant relationship between anxiety and depression and an increased in cancer pain intensity. They also found that participants' noncompliance with analgesics use was associated with anxiety and depression. Kwon et al. ${ }^{[24]}$ found a significant positive association between depression and pain intensity. Similarly, Edrington et al. ${ }^{[21]}$ reported a significant relationship between depression and poor cancer pain management. In other studies, a significant association has been reported between anxiety and patients' refusal to use analgesics; however, this association was not significant for depression. ${ }^{[19,38,39]}$

\subsection{Socio-demographic factors}

Socio-demographic factors shape the way cancer patient perceive cancer pain and influence their decisions toward pain management interventions, particularly age, gender, education, culture, and race.

Age. Closs et al. ${ }^{[38]}$ studied the influence of age on cancer pain and analgesics use in a sample of 90 patients in the UK . They found that participants more than 75 years old had significantly more barriers than those who were less than 60 years old. Beliefs about analgesics use and pain communication issues were the most significant barriers for older participants toward pain management. Moreover, age correlated positively with other barriers such as sensory and affective barriers. ${ }^{[28,32,33]}$ However, another study reported that younger patients had higher anxiety scores toward cancer pain management. ${ }^{[37]}$

Gender. Gender also influences perspectives of cancer patients toward pain management. In a study on 170 Turkish participants, male participants had more barriers than female participants. ${ }^{[20]}$ Male participants reported significant higher scores related to "communication barrier". In another study, a significant relationship has been reported between male gender and the desired to be a good patient or fear of distracting physicians from treating cancer. ${ }^{[28]}$ Based on gender differences, Simone et al. ${ }^{[26]}$ found that male participants' analgesics use was significantly less, while women had more fatalistic beliefs. ${ }^{[27]}$ In contrast, there were no differences between male and female Norwegian participants. ${ }^{[32]}$

Educational level. Cancer patients' education levels play an important role in developing barriers toward cancer pain management. Cancer patients with an educational level beyond high school have fewer barriers compared to those with less education. ${ }^{[26,30]}$ Haozous et al. ${ }^{[29]}$ reported that American Indians participants with less education had more misconceptions related to cancer pain management.

Culture. Cancer patients may develop misconceptions about pain management depending on their cultural background. An ethnographic qualitative research conducted to explore how American Indians who live in a community - oriented culture react towards analgesics in treating cancer pain. ${ }^{[29]}$ It has been found that participants believed that expressing pain was seen as a sign of weakness and complaining about cancer pain will only extend their vulnerability. ${ }^{[29]}$ The study participants reported aversion to report pain or take cancer pain management, as this may degrade their images among their community members. Similarly, in Taiwanese culture, cancer patients do not report cancer pain or accept using analgesics because they consider pain a necessary aspect of life. ${ }^{[25]}$ On the other hand, cultural differences among participants in the United States were examined and found that culture was not a significant determinant of cancer pain management. ${ }^{[27]}$

Race. Belonging to a specific racial group influences cancer patients' pain management. According to Simone et al., ${ }^{[26]}$ white Americans participants used more analgesics than all other races in their study. These findings were supported in another study in USA by Wilkie et al. ${ }^{[36]}$ who reported that participants who belong to a minority group (e.g. Hispanics) had significantly more barriers toward cancer pain management. ${ }^{[36]}$ A study in USA also compared the barriers between Hispanic and non - Hispanic cancer patients, found that race significantly acted as a barrier toward cancer pain management among study participants. ${ }^{[30]}$ 


\section{Discussion}

This literature review aimed to identify and explore adult cancer patients' barriers toward cancer pain management. Findings in this literature review will assist health care providers to identify these barriers and develop strategies that enhance cancer patients' ability to live without pain and improve their quality of life. This literature review showed the following patient' barriers toward cancer pain management: poor pain communication, fatalism, fear of physiological dependence, fear of drug side effects, affective barriers, and sociodemographic factors. We found that these themes can be classified into four categories (see Figure 3): a) cognitive barriers that include poor pain communication, fatalism, and fear of addiction and tolerance; b) sensory barrier that includes drug side effects; c) affective barriers that include anxiety and depression of cancer patients, and d) socio-demographic factors that include age, gender, educational level, culture, and race. We also concluded that each theme interacts or interconnects with other themes in the same or across different categories, and therefore, influences the effectiveness of cancer pain management accordingly. For example, literature reported women had more fatalistic beliefs. ${ }^{[32]}$ This shows that gender as a socio-demographic factor interconnects with fatalism that falls under cognitive barriers.

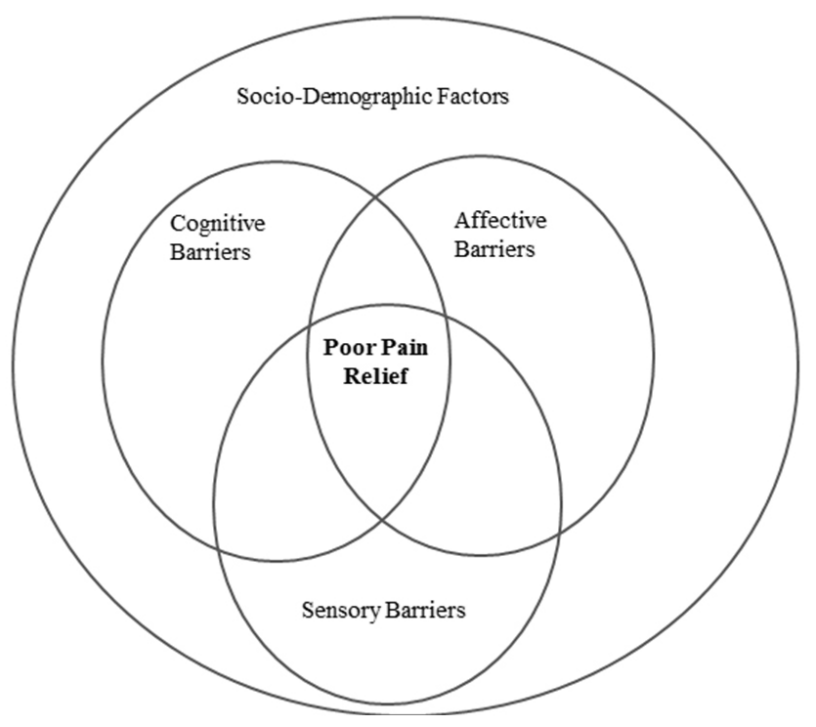

Figure 3. Adult cancer patients' barriers related to pain management

\subsection{Cognitive barriers}

Cognitive barriers (poor pain communication, fatalism, and fear of physiological dependence) may act as barriers toward the effectiveness of cancer pain management. Our findings suggest that poor communication is a significant barrier to pain management. Fear of distracting physicians from treating cancer and desire to be good patients are two concepts

Published by Sciedu Press that we reported in this literature review are in consistent with other research that suggest cancer patients who desire to be good patients believe that vocalizing pain is a sign of weakness.. ${ }^{[40]}$ Tung et al. ${ }^{[40]}$ reported that Chinese patients value stoicism, which refers to experience happiness or pain without showing emotions. Therefore, health care providers in Chinese communities may manage pain inadequately. This corresponds with the findings of Grant et al. ${ }^{[41]}$ who reported that participants tend not to report their cancer pain even when it is severe because complaining of pain was perceived as a weakness. The belief that good patients do not complain is influenced by patients' cultural and social norms as we reported in in this literature review.

In contrast to our findings, a study by Shinjo et al. ${ }^{[42]}$ found that participants reported their pain and asked for cancer pain management because of their desire to restore their quality of life and their perceptions that good death should happen without pain.

In our findings we described how patients avoided reporting pain based on their different beliefs, other studies found that reporting pain is important to their study participants. However, there are other factors that compromised the effectiveness of cancer pain management. For example, ineffective pain communication between healthcare providers and patients was linked to patients' beliefs of inadequate pain control. ${ }^{[6]}$ Lack of information related to cancer pain management or insensitive views of health care professionals to cancer pain experiences reduced patients' communication of their pain relief needs. ${ }^{[6]}$ Others reported that lack of communication between health care providers and cancer patients was the reason for patients' non-adherence, reluctance, or refusal of cancer pain treatment. ${ }^{[43]}$

The term fatalism constantly emerged in the literature as a significant patient's barrier toward cancer pain management. Fatalism is "the doctrine that what will be will be, or that human action has no influence on events". ${ }^{[4]}$ Fatalism for cancer patients means that cancer pain is inevitable, which means that pain is part of the disease; therefore, cancer patients might be reluctant to use analgesics for something that is unavoidable in their condition. Fatalistic beliefs were found to be more common among females, minority populations (e.g. Hispanics), and among cancer patients with less education. Fatalistic beliefs related to pain management were also reported in other studies concerning cancer pain meaning. ${ }^{[41,45]}$ These studies reported that participants viewed cancer pain as a natural pathological process of cancer and labeled cancer pain as an indicator of decreasing the chances of survival or nearing death. The literature also revealed also that some cancer patients had religious fatalistic beliefs that 
pain is from God. These religious fatalistic beliefs may be found in Islam, Hinduism, or Buddhism. A study that examined Buddhist cancer patients supported our findings. ${ }^{[40]}$ In Buddhism, to live is to suffer through life. Buddhist patients believe that pain is part of life and their pain is because of bad actions in their past, so they tend to refuse pain treatment to accept their own Karma. ${ }^{[40]}$ This shows how fatalism influence patients' decisions in enduring pain and refusing pain management.

Pathmawathi et al. ${ }^{[6]}$ reported contrasting results related to fatalism in Malaysia who found that while participants were having severe pain, they maintained their hope and improved their coping through their religious fatalistic beliefs. Religious fatalism increased the pain coping mechanisms for cancer patients. Some participants believed that prayers, mediation, reciting Quran verses, or drinking from holy water would help in relieving pain and calming their distress. This indicates that fatalism for some cancer patients is a complementary element or a non-pharmacological strategy than a barrier to cancer pain management.

Fear of addiction and tolerance are two types of physiological dependence barriers that constantly emerged in our literature review. Our finding was supported by Grant et al. ${ }^{[41]}$ who found that media and political spheres widely influenced the perspectives of Australian cancer patients toward morphine use. Patients believed that morphine cause addiction or tolerance, which directly affected the use and acceptance of morphine for cancer pain management among Australians. In a quite similarity, physicians in ten Asian countries found to believe that opioids usage for treating cancer pain would lead to addiction among this patient population. ${ }^{[46]}$ This indicates that there is a global fear of developing addiction from opioids use.

The concerns of addiction and tolerance of using cancer pain management in our literature review were inconsistent with the findings of other studies. For example, in Shinjo et al's. ${ }^{[42]}$ Study participants believed that the severity and impacts of cancer pain on their quality of life is more important than their fear of addiction. The participants' concerns of addiction or tolerance were eliminated because of the constant monitoring by nursing staff with appropriate opioids' weaning plan corresponding with their pain scores. In agreement, Shinjo et al. ${ }^{[42]}$ emphasized that their study participants showed acceptance and adherence to cancer pain management when health care providers explained the remote chances to develop addiction or tolerance with cancer pain management. This highlights that cancer patients' concerns of physiological dependence can be modified for better pain management outcomes. Effective education about can- cer pain management showed positive impacts on reducing cancer patients' fears and increasing their adherence to analgesics. $^{[43]}$

\subsection{Sensory barrier}

We found in several studies included in our literature review that fear of drug side effects, such as nausea, drowsiness, and saying or doing embarrassing things are common barriers among cancer patients receiving pain analgesics. Likewise, Torresan et al. ${ }^{[4]}$ reported the presence of a relationship between the recurrence of drug side effects among study participants and their reluctance or refusal to use analgesics. This created a barrier among study participants to continue the prescribed cancer pain medication. Moreover, Shinjo et al. ${ }^{[42]}$ reported that study participants who had relatives or friends that suffered from drug side effects tended to refuse analgesics, however, other participants accepted analgesics, despite knowing related side effects, when health care providers assured them that pain medication would be discontinued if side effects occur. It seems that the strong desire of cancer patients to have better quality of life with no pain increased their acceptance to use analgesics. ${ }^{[42,43]}$

\subsection{Affective barriers}

Our literature review showed that anxiety and depression are two affective barriers that may reduce the effectiveness of cancer pain management. These two barriers could have weak to severe impacts on cancer patients' attitudes toward adhering to analgesics. Similar results to our findings have been reported in a study of bereaved families' experiences with cancer pain and opioids use. ${ }^{[43]}$ The study participants reported that their relatives were anxious about using opioids for pain control. This directly affected their decisions to accept opioids to treat their cancer pain, which resulted in severe episodes of pain. ${ }^{[43]}$ Another study also supported these findings and reported that some cancer patients who experienced depression or anxiety because of cancer, refused or forgot to take analgesics for their cancer pain. ${ }^{[8]}$

However, contrary results were reported in a study that examined palliative patients' perceptions with breakthrough pain. ${ }^{[6]}$ Participants who felt helpless, hopeless, and wished to die, were relieved after using analgesics. This shows that cancer pain may induce anxiety and depression among cancer patients, and effective pain management might relieve these symptoms. In a qualitative study, depression was common among study participants, but this did not influence participants' decisions in accepting analgesics. ${ }^{[47]}$ The study participants showed adherence to cancer pain management, despite of their depression. 


\subsection{Socio-demographic factors}

Socio-demographic factors reported in our literature review as cancer patients' barriers toward pain management. The main factors that we identified are: age, gender, education, culture, and race. We explored in the literature the differences between older adults and younger adults in reporting cancer pain, accepting analgesics use, and adhering to pain treatment. We found that older adults have experienced more concerns than younger adults toward cancer pain management. This is in accord with the findings by Makris et al. ${ }^{[48]}$ who interviewed patients aged 65 years old and above with back pain. The study participants showed negative attitudes toward seeking treatment for their pain because of their beliefs that back pain is part of being old. Participants also did not report back pain to their health care providers because they wanted their physicians to focus on their comorbidities not on their back pain. Participants also did not adhere to pain medication because they have concern of addiction or negative interactions with their other co-morbidities medication. Similarly, we found that increased age was associated with more concerns about addiction and using analgesics. On the contrary, in a qualitative descriptive study, ${ }^{[8]}$ older adults adhered to analgesics to maintain the sense of control of their life. The participants were receiving palliative care and found themselves helpless with severe levels of pain, which caused them to accept analgesics to restore their abilities to control their life. ${ }^{[8]}$

The influence of gender differences on cancer patents' adherence to pain management is another barrier reported in our literature review. Majority of the included studies in our literature review reported that male participants had more barriers than females, while few studies showed no significant differences between male and female participants related to adhering to cancer pain management. Our findings are supported by Chou at al's. ${ }^{[49]}$ study who compared gender differences with the hesitancy to use analgesics, adherence to analgesics, and effectiveness of pain management among Taiwanese cancer patients. They found that female participants were more concerned with using analgesics and $74 \%$ of female participants had significantly higher beliefs that analgesics should not be taken around the clock than male participants $(51 \%)$. Similarly, Samulowitz et al. ${ }^{[50]}$ found that men in chronic pain presented more stoics, and avoided seeking health care, while women were more willing to report their pain and ask for analgesics. However, the researchers reported that the women felt that their chronic pain was down-valued and undertreated by their health care providers. These findings are comparable to our findings, where male participants of most included studies in our literature review had more barriers than female participants

Published by Sciedu Press toward cancer pain management. These findings indicate that gender's roles and responsibilities influence how cancer patients perceive cancer pain management.

Cancer patients' educational levels have been reported in our literature review as a factor that influences patients' decisions in accepting cancer pain management. We found that higher level of education was associated with less adult cancer patients' barriers. Similar findings were found by Emanuel et al. ${ }^{[51]}$ that education level influences how people understand and process the health information in order to identify the aspects of health literacy needed to their condition.

Cultural background influences how adult cancer patients view their pain management. We found in our literature review that cancer patients' cultures influenced their perceptions related to cancer pain management. This was supported by Kwok et al's. ${ }^{[52]}$ systematic review, who found that there is evidence for cultural differences in experiencing pain, having fatalistic beliefs about cancer pain, and accepting pain management. Equally, we showed in this literature review that cancer patients' culture shape and interpret the meaning of cancer pain, and determine how to report pain to others. This meaning is identified as the nature of the perceived relationship between patients and their world. Therefore, cancer patients' cultural beliefs normalize or problematize the pain experience. Cancer pain as a problematic situation was found in a study by Tung et al. ${ }^{[40]}$ The authors reported that many Chinese people refuse to discuss their pain because they belief that talking about their pain to someone outside of their family will bring the shame to every member in the family, which causes health care providers to face difficulties in assessing and managing their pain effectively. This supported by Haozous et al.'s ${ }^{[29]}$ study included in our literature review who found that some adult cancer patients refused analgesic, such as American Indians, because of their fears it will degrade their images among their community; especially if they complain about pain. Cultural beliefs varied from one patient population to another; nevertheless, it has a significant impact on the effectiveness of cancer pain management.

We also reported in our review that being a member of certain racial group is considered a barrier among cancer patients who require pain management. Similar results have been reported in another literature review by Kwok et al. ${ }^{[52]}$ who found that even in one society each group of people had different norms for perceiving and reporting cancer pain; African Americans, Hispanics, and Asian Americans reported different barriers toward cancer pain management compared to white Americans. Some black Caribbean patients also believed their relief of pain would come only after 
they die. ${ }^{[52]}$ Race also has been studied as a barrier toward pain management from the perspective of socioeconomic status and access to services. Racial minorities are less likely to receive effective cancer pain management because of their less income, lack of health insurance, and inadequate access to health care services. ${ }^{[52]}$ This shows that race plays an important role as a social determinant of health, which influences the effectiveness of cancer pain management.

In summary, various cancer patients' barriers that belong to different categories interchangeably influenced the effectiveness of pain management. These barriers often interacted with each other resulting in further complications of pain management efforts. Our literature review expanded the identified cancer patients' barriers reported in previous literature and how they interact with each other was found to be a key for proper management of pain among this patient population. This contributes to better understanding of these barriers and how to combat them. Findings in this literature review identify the need to raise awareness among clinicians and cancer patients about these barriers in order to reach to adequate pain management.

\subsection{Strengths and limitations}

It is important to acknowledge the strengths and limitation of this literature review. One of the main strengths of this literature review is the focus on cancer patients' barriers without including organization or health care professionals' barriers to reduce the complexity of the issue of the barriers toward cancer pain management. Another strength is that all studies included in this literature review are primary studies published within the last ten years. Additionally, the included articles studied various cancer patients' perspectives with different backgrounds and ethnicities, such as westerns, Asians, Africans, and people from Middle East. Moreover, the majority of included articles used validated and reliable tools and presented their findings while maintaining the rigor of their studies.

Few limitations were found in this literature review. The majority of included articles were quantitative studies (19), two qualitative studies, and none used the mixed methods approach. Another limitation is that quantitative studies included in this literature review used descriptive designs; convenient sampling and some studies had a small sample size. These might affect the generalizability of their findings. However, generalizability might not be an issue when exploring the phenomenon of cancer patients' barriers toward pain management because the investigators studied the subjec- tive experiences of cancer patients toward pain management, which can be unique according to the context of the study setting.

\subsection{Implications and recommendations}

This literature review highlighted the complexity of adult cancer patients' barriers toward pain management. Therefore, it is essential to increase the awareness about this issue among health care providers and cancer patients. It is recommended to develop educational activities that include materials for oncology nurses to enhance their competency in identifying patients' barriers and build strategies to eliminate these barriers. Educational activities by nurses will be beneficial for cancer patients who have barriers toward pain management to correct their misconceptions about cancer pain and its management. Cancer patients who had adequate knowledge about opioid painkillers had no concerns of using analgesics or adhering to prescribed cancer pain management. ${ }^{[41,42]} \mathrm{A}$ research is also recommended to explore other barriers of cancer pain management, such as organization or health care providers' barriers.

\section{Conclusion}

Adult cancer patients' barriers toward pain management significantly compromise the effectiveness of pain management and affect cancer patients' quality of life. The main barriers that have been reported in this literature include cognitive barriers that include cancer patients' perspective about pain and its management. These barriers include poor pain communication, fatalism, and fear of physiological dependence. Sensory barrier is also reported in this literature review, which includes cancer patients' fears of drug side effects. Cancer patients' emotional disorders, such as anxiety and depression, are two affective barriers toward cancer pain management. Lastly, socio-demographic factors, such as age, gender, educational level, culture, and race, were found to influence pain management among adult individuals with cancer. A better understanding of cancer patients' barriers toward pain management by healthcare providers will result in better assessment of these barriers and better planning for effective pain treatment. This literature review includes suggestions of important implications that will enhance the knowledge and the practice of health care professionals regarding cancer pain management, such as educational activities and primary research.

\section{CONFlicts OF INTEREST Disclosure}

The authors declare that there is no conflict of interest. 


\section{REFERENCES}

[1] World Health Organization. Cancer. 2019. Available from: https : //www.who.int/cancer/en/

[2] Christo P, Mazloomdoost D. Cancer Pain and Analgesia. Annals of the New York Academy of Sciences. 2008; 1138: 278-298. PMid:18837907 https://doi.org/10.1196/annals.1414.03 3

[3] Kim EB, Han HS, Chung JH, et al. The Effectiveness of a SelfReporting Bedside Pain Assessment Tool for Oncology Inpatients. Journal of Palliative Medicine. 2012; 15: 1222-1233. PMid:22974435 https://doi.org/10.1089/jpm.2012.0183

[4] Rau KM, Chen JS, Wu HB, et al. The impact of pain control on physical and psychiatric functions of cancer patients: a nation-wide survey in Taiwan. Japanese Journal of Clinical Oncology. 2015; 45: 10421049. PMid:26292698 https://doi .org/10.1093/jjco/hyv12 4

[5] Pergolizzi JV, Gharibo C, Ho K. Treatment Considerations for Cancer Pain: A Global Perspective. Pain Practice. 2015; 15: 778-792. PMid:25469726 https : //doi .org/10.1111/papr.12253

[6] Pathmawathi S, Beng TS, Li LM, et al. Satisfaction with and Perception of Pain Management among Palliative Patients with Breakthrough Pain: A Qualitative Study. Pain Management Nursing. 2015; 16: 552-560. PMid:26256219 https://doi.org/10.1016/j.pm n. 2014.10.002

[7] Mehta A, Chan LS. Understanding of the concept of "Total pain": a prerequisite for pain control. Journal of Hospice \& Palliative Nursing. 2008; 10: 26-34. https ://doi .org/10.1097/01. NJH.0000306 $714.50539 .1 \mathrm{a}$

[8] Benedetti F, Thoen W, Blanchard C, et al. Pain as a reward: changing the meaning of pain from negative to positive co-activates opioid and cannabinoid systems. Pain. 2013; 154: 361-367. PMid:23265686 https://doi.org/10.1016/j.pain.2012.11.007

[9] Hackett J, Godfrey M, Bennett MI. Patient and caregiver perspectives on managing pain in advanced cancer: A qualitative longitudinal study. Palliative Medicine. 2016; 30: 711-719. PMid:26847524 https://doi.org/10.1177/0269216316628407

[10] Rouahi N, Zouhdi M. Arabic Tools for Assessment of Multidimensions of Pain and Discomfort Related to Cancer. Asian Pacific Journal of Cancer Prevention: Apjcp. 2016; 17: 2619-24.

[11] Carlson CL. Effectiveness of the World Health Organization cancer pain relief guidelines: an integrative review. Journal of Pain Research. 2016; 9: 515-534. PMid:27524918 https://doi.org/10.2147/ JPR.S97759

[12] Mercadante S, Roila F, Berretto O, et al. Prevalence and treatment of cancer pain in Italian oncological wards centres: a crosssectional survey. Supportive Care in Cancer. 2008; 16: 1203-1211. PMid:18478276 https://doi.org/10.1007/s00520-008-045 6-7

[13] Jacobsen R, Moldrup C, Christrup L, et al. Patients-related barriers to cancer pain management: a systematic exploratory review. Scand J Caring Sci. 2009; 23: 190-208. PMid:18785917 https://doi.org/10.1111/j.1471-6712.2008.00601.x

[14] Makhlouf M, Pini S, Ahmed S, et al. Managing pain in people with cance. A Systematic review of the attitudes and knowledge of professionals, patients, caregivers and public. Journal of Cancer Education. 2020; 214-240. PMid:31119708 https ://doi .org/10.1007/s1 3187-019-01548-9

[15] Cronin P, Ryan F, Coughlan M. Undertaking a literature review: A step-by-step approach. British Journal of Nursing. 2008; 17(1): 3843. PMid:18399395 https://doi.org/10.12968/bjon. 2008.1 7.1 .28059

Published by Sciedu Press
[16] Moher D, Liberati A, Tetzlaff J, et al. Preferred Reporting Items for Systematic Reviews and Meta-Analyses. PLoS Medicine. 2009; 6(7): e1000097. PMid:19621072 https://doi.org/10.1371/journa 1.pmed. 1000097

[17] Hong QN, Pluye P, Fabregues S, et al. Mixed Methods Appraisal Tool (MMAT), version 2018. Registration of Copyright (\#1148552), CanadianIntellectual Property Office, Industry Canada.

[18] Braun V, Clarke V. Thematic Analysis. Thematic Analysis. In H Cooper P, Camic D, Long A, Panter D, Rindskopf K. APA handbooks of research methods in psychology. Research design: Quantitative, qualitative, neuropsychological, and biological. APA handbooks in psychology. 2012; 57-71. PMid:22108560 https ://doi .org/10 $.1037 / 13620-004$

[19] Jacobsen R, Samsanaviciene J, Liuabarskiene Z, et al. Barriers to pain management among Lithuanian cancer patients. Pain Practice. 2010; 10: 145-157. PMid:20070553 https://doi.org/10.1111/ j.1533-2500.2009.00333.x

[20] Bagcivan G, Tosun N, Komurcu S, et al. Analysis of patient-related barriers in cancer pain management in Turkish patients. Journal of Pain \& Symptom Management. 2009; 38: 727-37. PMid:19692202 https://doi.org/10.1016/j.jpainsymman.2009.03.004

[21] Edrington J, Sun A, Wong C, et al. Barriers to pain management in a community sample of Chinese American patients with cancer. Journal of Pain \& Symptom Management. 2009; 37: 665675. PMid:19004613 https://doi.org/10.1016/j.jpainsym man.2008.04.014

[22] Liu Q, Gao LL, Dai YL, et al. Breakthrough Pain: A Qualitative Study of Patients with Advanced Cancer in Northwest China Pain Management Nursing. 2018; 19: 506-515. PMid:29506898 https://doi.org/10.1016/j.pmn.2017.11.006

[23] Saifan A, Bashayreh I, Batiha AM, et al. Patient- and family caregiverrelated barriers to effective cancer pain control. Pain Management Nursing. 2015; 16: 400-410. PMid:26025799 https ://doi .org/ $10.1016 / j \cdot$ pmn. 2014.09.007

[24] Kwon JH, Hui D, Chisholm G, et al. Experience of barriers to pain management in patients receiving outpatient palliative care. Journal of Palliative Medicine. 2013; 16: 908-914. PMid:23758527 https://doi.org/10.1089/jpm.2012.0610

[25] Chou PL, Rau KM, Lin CC. Development and psychometric testing of a short version of the Barriers Questionnaire-Taiwan form for cancer patients. International Journal of Nursing Studies. 2011; 48: 1071-1079. PMid:21396643 https://doi.org/10.1016/j. ijnurstu.2011.02.006

[26] Simone C, Vapiwala N, Hampshire M, et al. Cancer Patient Attitudes toward Analgesic Usage and Pain Intervention. The Clinical Journal of Pain. 2012; 28: 157-162. PMid:21705874 https: //doi.org/10.1097/AJP.0b013e318223be30

[27] Kwon JH, Oh SY, Chisholm G, et al. Predictors of high score patientreported barriers to controlling cancer pain: a preliminary report. Supportive Care in Cancer. 2013; 21: 1175-1183. PMid:23151648 https://doi.org/10.1007/s00520-012-1646-x

[28] Gunnarsdottir S, Kaasa S, Klepstad P, et al. A multicenter study of attitudinal barriers to cancer pain management. Supportive Care in Cancer. 2017; 25: 3595-3602. PMid:28653107 https://doi .org/ 10.1007/s00520-017-3791-8

[29] Haozous EA, Knobf MT. "All my tears were gone": suffering and cancer pain in Southwest American Indians. Journal of Pain \& Symptom Management. 2013; 45: 1050-1060. PMid:22940564 https://doi.org/10.1016/j.jpainsymman.2012.06.001

[30] Stein KD, Alcaraz KI, Kamson C, et al. Sociodemographic inequalities in barriers to cancer pain management: a report from the American Cancer Society's Study of Cancer Survivors-II (SCS-II). 
Psycho-Oncology. 2016; 25: 1212-1221. PMid:27421683 https: //doi.org/10.1002/pon.4218

[31] Alexopoulos EC, Koutsogiannou P, Moratis E, et al. Pain in cancer patients: The Greek experience. European Journal of Oncology Nursing. 2011; 15: 442-446. PMid:21093370 https ://doi .org/10.1 016/j.ejon.2010.10.008

[32] Valeberg BT, Hanestad BR, Klepstad P, et al. Cancer patients' barriers to pain management and psychometric properties of the Norwegian version of the Barriers Questionnaire II. Scandinavian Journal of Caring Sciences. 2008; 23: 518-528. PMid:19055592 https://doi.org/10.1111/j.1471-6712.2008.00639.x

[33] Al-Atiyyat NM, Vallerand AH. Patient-related attitudinal barriers to cancer pain management among adult Jordanian patients. European Journal of Oncology Nursing. 2018; 33: 56-61. PMid:29551178 https://doi.org/10.1016/j.ejon.2018.01.009

[34] Liang SY, Tung HH, Wu SF, et al. Concerns About Pain and Prescribed Opioids in Taiwanese Oncology Outpatients. Pain Management Nursing. 2013; 14: 336-342. PMid:24315256 https: //doi.org/10.1016/j.pmn.2011.08.004

[35] Lim SN, Han HS, Lee KH, et al. A Satisfaction Survey on Cancer Pain Management Using a Self-Reporting Pain Assessment Tool. Journal of Palliative Medicine. 2015; 18: 225-231. PMid:25650504 https://doi.org/10.1089/jpm.2014.0021

[36] Wilkie D, Ezenwa MO, Yao Y, et al. Pain Intensity and Misconceptions Among Hospice Patients With Cancer and Their Caregivers: Status After 2 Decades. American Journal of Hospice and Palliative Medicine. 2017; 34: 318-324. PMid:27006391 https: //doi.org/10.1177/1049909116639612

[37] Jacobsen R, Samsanaviciene J, Liubarskiene Z, et al. Barriers to cancer pain management in Danish and Lithuanian patients treated in pain and palliative care units. Pain Management Nursing. 2014; 15: 51-8. PMid:24602424 https://doi.org/10.1016/j.pmn.2012 .06 .002

[38] Closs SJ, Chatwin J, Bennett MI, et al. Cancer pain management at home (II): does age influence attitudes towards pain and analgesia? Supportive Care in Cancer. 2009; 17: 781-786. PMid:19066984 https://doi.org/10.1007/s00520-008-0548-4

[39] Jacobsen R, Moldrup C, Christrup L, et al. The Danish Barriers Questionnaire-II: preliminary validation in cancer pain patients. Pain Practice. 2009; 9: 266-274. PMid:19549059 https ://doi .org/10 $.1111 / j .1533-2500.2009 .00296 . x$

[40] Tung W, \& Li Z.. Pain Beliefs and behaviors among Chinese. Home Health Care Management \& Practice. 2015; 27(2): 95-97. https://doi.org/10.1177/1084822314547962

[41] Grant M, Ugalde A, Vafiadis P, et al. Exploring the myths of morphine in cancer: Views of the general practice population. Supportive Care in Cancer. 2015; 23(2): 483-489. PMid:25138618 https://doi.org/10.1007/s00520-014-2379-9
[42] Shinjo T, Morita T, Hirai K, et al. Why people accept opioids: role of general attitudes toward drugs, experience as a bereaved family, information from medical professionals, and personal beliefs regarding a good death. Journal of Pain \& Symptom Management. 2015; 49(1): 45-54. PMid:24929030 https ://doi.org/10.1016/j.jp ainsymman.2014.04.015

[43] Webber K, Davies AN, Cowie MR. Disparities between Clinician and Patient Perception of Breakthrough Pain Control. Journal of Pain \& Symptom Management. 2016; 51(5): 933-937. PMid:26740387 https://doi.org/10.1016/j.jpainsymman.2015.12.309

[44] Merriam-Webster. Fatalism. Available from: https: //www.merriam-webster.com/dictionary/fatalism?s $\mathrm{rc}=$ search-dict-hed

[45] McPherson CJ, Hadjistavropoulos T, Lobchuk MM, et al. Cancerrelated pain in older adults receiving palliative care: patient and family caregiver perspectives on the experience of pain. Pain Research \& Management. 2013; 18: 293-300. PMid:23957019 https : //doi.org/10.1155/2013/439594

[46] Kim Y, Ahn J, Calimag M, et al. Current practices in cancer pain management in Asia: a survey of patients and physicians across 10 countries. Cancer Medicine. 2015; 4(8): 1196-120. PMid:25914253 https://doi.org/10.1002/cam4.471

[47] Torresan M, Garrino L, Borraccino A, et al. Adherence to treatment in patient with severe cancer pain: A qualitative enquiry through illness narratives. European Journal of Oncology Nursing. 2015; 19(4): 397-404. PMid:25691299 https://doi.org/10.1016/j . ejon.2015.01.001

[48] Makris U, Higashi R, Marks E, et al. Ageism, negative attitudes, and competing co-morbidities - why older adults may not seek care for restricting back pain: a qualitative study. BMC Geriatrics. 2015; 15(1): 1-14. PMid:25887905 https://doi.org/10.1186/s128 77-015-0042-z

[49] Chou P, Fang Y, Sun L, et al. Gender Difference in Cancer Patients' Adherence to Analgesics and Related Outcomes of Pain Management. Cancer Nursing. 2016; 41(6): E11-E18. PMid:28753193 https://doi.org/10.1097/NCC.0000000000000532

[50] Samulowitz A, Gremyr I, Eriksson E, et al. "Brave Men" and "Emotional Women": A Theory-Guided Literature Review on Gender Bias in Health Care and Gendered Norms towards Patients with Chronic Pain. Pain Research \& Management. 2018; 1-14. PMid:29682130 https://doi.org/10.1155/2018/6358624

[51] Emanuel A, Godinh A, Steinman C, et al. Education differences in cancer fatalism: The role of information-seeking experiences. Journal of Health Psychology. 2018; 23(12): 1533-1544. PMid:27553609 https://doi.org/10.1177/1359105316664129

[52] Kwok W, Bhuvanakrishna T. The Relationship Between Ethnicity and the Pain Experience of Cancer Patients: A Systematic Review. Indian Journal of Palliative Care. 2014; 20(3): 194-200. PMid:25191006 https://doi.org/10.4103/0973-1075.138391 